\title{
Quality of Life Is Worse at 6-10 Years after Radioactive lodine Treatment of Graves' Disease as Compared with Antithyroid Drugs or Surgery
}

\section{Sun Y. Lee}

Section of Endocrinology, Diabetes, and Nutrition; Department of Medicine, Boston University School of Medicine, Boston Medical Center, Boston, Massachusetts, U.S.A.

Review of: Törring O, Watt T, Sjölin G, Byström K, Abraham-Nordling M, Calissendorff J, Cramon PK, Filipsson Nyström H, Hallengren B, Holmberg M, Khamisi S, Lantz M, Wallin G 2019. Impaired quality of life after radioiodine therapy compared with antithyroid drugs or surgical treatment for Graves' hyperthyroidism: a long-term follow-up with the Thyroid-Related Patient-Reported Outcome Questionnaire and 36-Item Short Form Health Status Survey. Thyroid 29:322-331. PMID: 30667296.

\section{SUMMARY}

\section{Background}

Graves' disease is a common cause of hyperthyroidism and may be associated with a negative impact on patients' quality of life (QoL) $(1,2)$. It can be accompanied by extrathyroidal complications such as ophthalmopathy, dermopathy, and acropachy. Usual treatment options include antithyroid drugs (ATDs), radioiodine (RAI), or surgery. Previous studies have reported similar general-health QoL with the three treatment methods, but were limited to shorter-term follow up $(3,4)$. The current study was conducted to assess long-term QoL in patients with Graves' disease who have been treated in a routine clinical setting.

\section{Methods}

A cohort of 2916 patients with a new diagnosis of hyperthyroidism between 2003 and 2005 was established from seven centers in southern Sweden. Patients were treated according to usual clinical practice. From this cohort, 1186 patients with Graves' disease were recruited for the current study 6 to 10 years after the original diagnosis to assess QoL measures. Those with recurrent Graves' disease were excluded.
QoL was assessed using the validated Swedish versions of the Thyroid-Related Patient-Reported Outcome Questionnaire (ThyPRO; $\mathrm{n}=975$ ) and the general 36-Item Short Form Health Status Survey (SF-36; $n=964$ ) questionnaires completed by participants; scores from ThyPRO were compared with scores from a general population sample $(n=712)$. ThyPRO measures thyroid-related QoL, including physical and mental symptoms, impaired function, well-being, and negative impact on overall QoL. Lower scores in ThyPRO represent better health status. SF-36 measures general health status, including physical, mental, and social function and well-being. Higher scores on SF-36 represent better health status.

Participants were divided in three groups: (i) patients treated with ATDs only, (ii) patients treated with RAI (with or without previous treatment with ATD), but not surgery, and (iii) patients treated with subtotal or total thyroidectomy (with or without previous treatment with ATD or RAI). Age, sex, and presence of comorbidities (presence of diabetes type 1 or 2 , vitamin B12 deficiency, rheumatic disease, celiac disease, Addison's disease, vitiligo, psoriasis, sar- 
coidosis, and other chronic disease) were assessed to adjust for potential confounders.

\section{Results}

Patients were assessed at a mean $( \pm$ SD) of $8 \pm 0.9$ years after the diagnosis of Graves' disease. There were 347 patients (36\%) in the ATD group, 395 (41\%) in the RAI group, and 233 (24\%) in the surgery group. Median ages were significantly different for the treatment groups: 43 years (range, 33-55) for the ATD group, 54 years (range, 45-63) for the RAI group, and 35 years (range, 29-46) for the surgery group. The proportion with comorbidities was also significantly different among groups; the number of participants with comorbidities were 102 (29\%) in the ATD group, 173 (44\%) in the RAI group, and 76 (33\%) in the surgery group. Distribution of sex was similar among groups.

Overall, patients with treated Graves' disease had worse scores on thyroid-related QoL measures, as assessed by ThyPRO, than the general population. However, patients treated with ATD or surgery had scores of tiredness and emotional susceptibility that were similar to those of the general population. Among the three treatment groups, patients who received RAI had worse thyroid-specific QoL scores than patients treated with ATD or surgery, as measured by ThyPRO (score of 27 in RAI group, compared to 21 in the ATD group and 22 in the surgery group). The RAl group also had worse scores for goiter symptoms, hyperthyroid symptoms, tiredness, anxiety, depression, emotional susceptibility, impaired social life, impaired daily life, and impaired sex life than the ATD and surgery groups. In addition, the RAI group had worse scores in hypothyroid symptoms, eye symptoms, and appearance than the ATD group.

A similar pattern was found in general QoL measures as assessed with the SF-36 questionnaire. Patients treated with RAI had worse scores on all SF-36 scales than the ATD group, especially in the areas of general health, role limitations, and social function. Patients treated with RAl had worse scores than those who underwent surgery except in bodily pain and in the physical component summary.

\section{Conclusions}

Regardless of treatment method, patients with Graves' disease had worse QoL measures at 6 to 10 years after diagnosis than the general population. In this long-term analysis, there were greater impairments of both thyroid-specific and general QoL scores in those who received RAI than in patients treated with ATD or surgery. These findings should be further substantiated in future studies, given the potential long-term implications of selecting treatment choices for patients with Graves' disease.

\section{COMMENTARY}

This study (5) supports findings of previous studies $(1,2)$, which showed worse QoL scores in patients with Graves' disease than in the general population. However, findings of a worse long-term QoL in those treated with RAl, as compared with those treated with ATD or surgery, is in contrast to previous studies. A report of 174 patients with Graves' disease strat- ified by age and randomly assigned to ATD, surgery, or RAl treatment in Sweden had shown no significant differences in QoL at the 3-year follow-up (3). However, in that study, there were high rates of relapse in those treated with an ATD (42\% in the 20-34 age group and 34\% in the 35-55 group) and more frequent eye problems in those treated with 
RAl. Another long-term study of patients at 14 to 21 years after initial enrollment also did not show significant differences in QoL scores (4). However, at follow-up only $51 \%$ of the 147 patients had normal serum TSH levels, with $44 \%$ with low TSH levels and $4 \%$ with high TSH levels. Additionally, another randomized study of 308 patients showed no difference in general QoL measures, as assessed by SF-36, after ATD or RAI treatment at 4-year follow-up (6). Only those in whom thyroid ophthalmopathy developed had worse QoL, which was more frequent in the RAl-treated group than in the ATD-treated group (46\% and $27.6 \%$, respectively).

Strengths of the current study include a large sample size and long-term follow-up (mean, 8 years) after the initial diagnosis of Graves' disease. Limitations include differences in characteristics in the three groups, especially with regard to age and presence of comorbidities, and the lack of information on serum thyroid-function tests. Patients who were treated with RAl were generally older and more likely to have had comorbidities than those treated with ATD or surgery. Although age and presence of comorbidities were adjusted for the analyses, there may have been some selection bias for RAl treatment, such as a contraindication for surgery and suboptimal treatment response to or recurrence with an ATD alone. These patients may have had a more prolonged or complicated disease course, which could have contributed to worse QoL measures. As there was also no information available on thyroid hormone status at follow-up of these subjects, the potential effects of concurrent biochemical hypothyroidism or hyperthyroidism on QoL could not be assessed.

This study reports a new observation that there may be a negative impact of RAI treatment on long-term QoL measures in patients with Graves' disease. Further studies to ascertain this observation would be needed, given the potentially important impact of choosing treatment options for patients with Graves' disease in routine clinical practice.

\section{References}

1. Elberling TV, Rasmussen AK, Feldt-Rasmusen U, Hording M, Perrild H, Waldemar G 2004 Impaired health-related quality of life in Graves' disease: a prospective study. Eur J Endocrinol 151:549-555.

2. Watt T, Groenvold M, Rasmusen AK, Bonnema SJ, Hegedüs L, Bjorner JB, Feldt-Rasmussen U 2006 Quality of life in patients with benign thyroid disorders: a review. Eur J Endocrinol 154:501-510.

3. Ljunggren JG, Torring O, Wallin G, Taube A, Tallstedt L, Hamberger B, Lundell G 1998 Quality of life aspects and costs in treatment of Graves' hyperthyroidism with antithyroid drugs, surgery, or radioiodine: results from a prospective, randomized study. Thyroid 8:653-659.
4. Abraham-Nordling $M$, Torring $O$, Hamberger $B$, Lundell G, Tallstedt L, Calissendorff J, Wallin G 2005 Graves' disease: a long-term quality-of-life follow up of patients randomized to treatment with antithyroid drugs, radioiodine, or surgery. Thyroid 15:1279-1286.

5. Törring $O$, Watt T, Sjölin G, Byström K, AbrahamNordling M, Calissendorff J, Cramon PK, Filipsson Nyström H, Hallengren B, Holmberg M, Khamisi S, Lantz M, Wallin G. 2019. Impaired quality of life after radioiodine therapy compared with antithyroid drugs or surgical treatment for Graves' hyperthyroidism: a long-term follow-up with the Thyroid-Related PatientReported Outcome Questionnaire and 36-Item Short Form Health Status Survey. Thyroid 29:322-331. 
HYPERTHYROIDISM Quality of Life Is Worse 6-10 Years after Sun Y. Lee

Radioactive lodine Treatment of Graves' Disease as

Compared with Antithyroid Drugs or Surgery

6. Abraham-Nordling M, Wallin G, Traisk F, Berg G,

Calissendorff J, Hallengren B, Hedner P, Lantz M,

Nystrom E, Asman P, et al. 2010 Thyroid-associated

ophthalmopathy: quality of life follow-up of patients

randomized to treatment with antithyroid drugs or

radioiodine. Eur J Endocrinol 163:651-657.

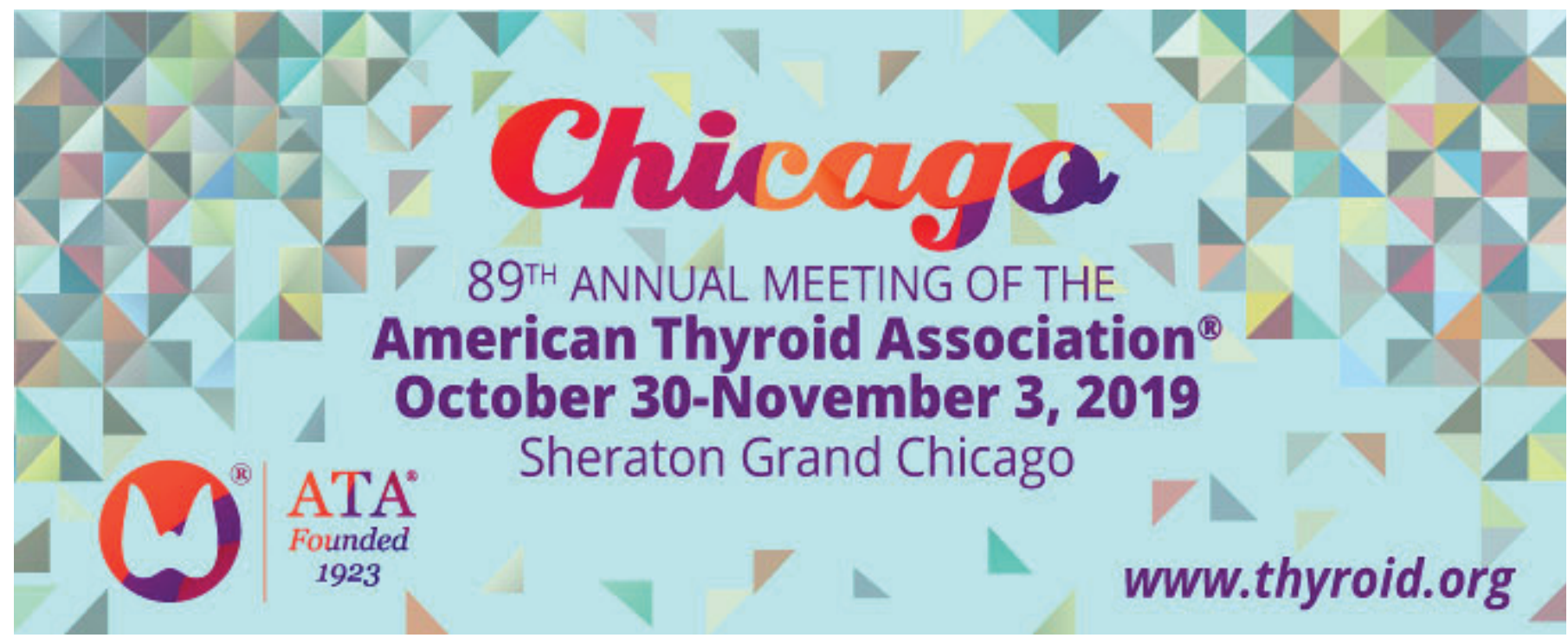

